IDDF2019-ABS-0017 CLINICO-MICROBIOLOGICAL PROFILE OF GASTRIC ASPIRATES AMONG PEDIATRIC PULMONARY TB CASES FROM A TERTIARY CARE HOSPITAL

Bineeta Kashyap*, Neha Gupta, Pooja Dewan, Puneeta Hyanki, Narendra Pal Singh. University College of Medical Sciences and Guru Teg Bahadur Hospital, Delhi, India

10.1136/gutjnl-2019-IDDFabstracts. 117

Background Childhood TB constitutes $10-20 \%$ of the total TB cases in high burden countries. The most common clinical features in pulmonary $\mathrm{TB}$ in children are non-productive cough, low fever and weight loss. Radiological features in pediatric pulmonary TB may also remain non-specific, most typical being hilar or paratracheal lymphadenopathy. So the diagnosis remains difficult. Microbiological confirmation remains challenging due to suboptimal pulmonary specimens in such cases. This study was aimed to analyze the clinicomicrobiological profile of gastric aspirate in pediatric pulmonary TB.

Methods Clinically suspected pediatric pulmonary TB cases $\leq 12$ years of age were recruited over a period of 1 year. Gastric aspirates were processed for microbiological diagnosis. As per Revised National Tuberculosis Control Program definitions, the cases were clinically diagnosed (clinical history and Mantoux test and/or contact history and/or radiological evidence) or microbiologically confirmed $[\geq 1$ of smear/culture/Cartridge Based Nucleic Acid Amplification Test (CBNAAT)] to be pediatric pulmonary TB.

Results Mean age of children in the study was 5.8 years. Of 27 cases, Mantoux test was reactive in 13 (48\%), 13(48\%) had a history of contact with an infectious case and BCG scar was present in $22(81.5 \%)$ cases. Fever was the predominant symptom followed by loss of appetite, weight loss and cough. Only one case did not have suggestive chest radiography while $18(66.7 \%)$ showed consolidation. Microbiological diagnosis could be established in $20(74 \%)$ cases, 8 (29.6\%) being smear positive, $12(44.4 \%)$ culture positive and 17 (63\%) positive by CBNAAT.

Conclusions Pediatric pulmonary TB remains difficult to diagnose due to the non-specific presentation, suboptimal samples and paucibacillary nature of the disease. Though microbiological confirmation by conventional tests remains low, using CBNAAT marginally increases the positivity. An integrated approach based on history, Mantoux test, radiology and microbiology increase chances of reaching an early diagnosis. Further research is needed for newer techniques to detect and manage paucibacillary disease in children at the earliest.

\section{IDDF2019-ABS-0021 DIAGNOSTIC COLONOSCOPY FOR DIVERTICULAR DISEASE IN UB SONGDO HOSPITAL}

${ }^{1}$ Bayasgalan Luvsandagva*, ${ }^{1}$ Battulga Adiyasuren, ${ }^{1}$ Narantsatsralt Jalbuu, ${ }^{1}$ Namsrai Renchinsengee, ${ }^{2}$ Davaadorj Duger. 'Ulaanbaatar Songdo Hospital, Mongolia; ${ }^{2}$ Mongolian National University of Medical Science, Mongolia

\subsection{6/gutjnl-2019-IDDFabstracts. 118}

Background Colonic diverticulum rises on blood vessel penetration site of colonic smooth muscle through the herniation process. Commonly asymptomatic and found incidentally during colonoscopy. But it could turn in to life-threatening disease once bleeding, inflammation or perforation occurs. The aims of this study were to detect the incidence of complicated diverticular disease and diagnostic accuracy of colonoscopy in diverticular disease compared with abdominal contrastenhanced computed tomography (CT).

Methods This retrospective study included a total of 321 patients with colonic diverticulum diagnosed by lower gastrointestinal endoscopy within the past $\underline{8}$ years in Gastroenterology and Endoscopy department of Ulaanbaatar Songdo Hospital, Ulaanbaatar city, Mongolia. The borderline between the proximal and distal colon was splenic flexure. Chi-square and student t-test were used to differentiate the two groups. $\mathrm{P}>0.05$ was statistically significant.

Results The mean age of patients with colonic diverticulosis $58.67 \pm 14.45$ and incident of diverticulosis increased in elderly patients $(\mathrm{t}=-4.439 ; \mathrm{P}<0.001)$ and female were predominant $(\lambda 2=78.82 ; \mathrm{P}<0.001)$. Diverticulitis is a common complication and more frequently in patients with distal diverticulosis group $(\lambda 2=9.97 ; \mathrm{P}<0.001)$, diverticular bleeding more frequently in patients with proximal diverticulosis $(\lambda 2=13.49 ; \mathrm{P}<0.001)$ (table 1). Age, gender, complicated diverticular diseases divided by location of colonic diverticulosis is shown in table 2 . Distal diverticulosis was predominant in female patients group and proximal diverticulosis was predominant in male patients group (table 2). A total number of 79 patients were examined by both colonoscopy and CT together in approximate time. But $72.2 \%$ of results have missed the diverticulum and $22.2 \%$ of diverticulitis cases were misdiagnosed by suspected colonic cancer.

Conclusions The incidence of complicated diverticular disease around $6.5 \%$ in patients with colonic diverticulosis. The diverticulitis occurs more frequently than diverticular associated bleeding. The colonoscopy has higher diagnostic accuracy for colonic diverticulosis and complicated diverticular disease compared with abdominal contrast-enhanced computed tomography.

\section{IDDF2019-ABS-0022 THE EFFICACY OF PENTOXIFYLLINE ON ACUTE PANCREATITIS}

Dhanusorn Wanichagool*, Karjpong Techathuvanan. Division of Gastroenterology, Department of Medicine, Faculty of Medicine, Vajira Hospital, Navamindradhiraj University, Bangkok, Thailand

\subsection{6/gutjnl-2019-IDDFabstracts. 119}

Background Acute pancreatitis is an inflammatory condition that occurs in the pancreatic parenchyma and systemically affect other organs. The first phase of the disease, usually during the first week, is caused by the patient's systemic inflammatory response elicited by acinar cell injury. During this phase, the severity of acute pancreatitis is directly related to extrapancreatic organ failure, and several intercellular signaling proteins such as tumor necrosis factor-a (TNF-a) are involved. Although there is currently no specific treatment for such condition, there is evidence in animals model showing that the administration of pentoxifylline, a competitive nonselective phosphodiesterase inhibitor, is able to reduce inflammation 